\title{
The Green-Kubo formula for the spin-fermion system
}

\author{
V. Jakšić ${ }^{1}$, Y. Ogata ${ }^{2,3}$, C.-A. Pillet ${ }^{2}$ \\ ${ }^{1}$ Department of Mathematics and Statistics \\ McGill University \\ 805 Sherbrooke Street West \\ Montreal, QC, H3A 2K6, Canada \\ ${ }^{2}$ CPT-CNRS, UMR 6207 \\ Université du Sud, Toulon-Var, B.P. 20132 \\ F-83957 La Garde Cedex, France \\ ${ }^{3}$ Department of Mathematical Sciences \\ University of Tokyo \\ Komaba,Tokyo,153-8914 Japan
}

September 22, 2005

\begin{abstract}
The spin-fermion model describes a two level quantum system $\mathcal{S}$ (spin $1 / 2$ ) coupled to finitely many free Fermi gas reservoirs $\mathcal{R}_{j}$ which are in thermal equilibrium at inverse temperatures $\beta_{j}$. We consider non-equilibrium initial conditions where not all $\beta_{j}$ are the same. It is known that, at small coupling, the combined system $\mathcal{S}+\sum_{j} \mathcal{R}_{j}$ has a unique non-equilibrium steady state (NESS) characterized by strictly positive entropy production. In this paper we study linear response in this NESS and prove the Green-Kubo formula and the Onsager reciprocity relations for heat fluxes generated by temperature differentials.
\end{abstract}




\section{Introduction}

This is the third in a series of papers dealing with linear response theory in quantum statistical mechanics. In the first two papers in the series [JOP1, JOP2] we have given an abstract axiomatic derivation of the Green-Kubo formula for the heat fluxes generated by temperature differentials. In this paper we verify that this axiomatic derivation is applicable to the spin-fermion model (abbreviated SFM).

The Green-Kubo formula is one of the pillars of non-equilibrium statistical mechanics and is discussed in many places in physics literature (see e.g. [KTH]). A mathematical justification of this formula is one of the outstanding open problems in mathematical physics [Si]. In the literature, most existing results concern currents induced by mechanical driving forces such as time-dependent electric or magnetic fields (see [NVW, GVV, BGKS] for references and additional information). In contrast, there are very few results dealing with fluxes generated by thermodynamical driving forces such as temperature differentials. The central difficulty is that a mathematically rigorous study of linear response to thermodynamical perturbations requires as input a detailed understanding of structural and ergodic properties of non-equilibrium steady states (NESS). In the papers [JOP1, JOP2] we have bypassed this difficulty by assuming the necessary regularity properties as axioms. The general axiomatic derivation of the Green-Kubo formula in [JOP1, JOP2] has led to some new insights concerning the mathematical structure of non-equilibrium quantum statistical mechanics. Concerning applications to concrete models, it reduced the proof of the Green-Kubo formula to the study of regularity properties of NESS.

In most cases, the study of NESS of physically relevant models is beyond existing mathematical techniques. The information necessary to study linear response theory has been obtained only recently and only for a handful of models [JP2, JP3, AH, AP, FMU]. To the best of our knowledge the SFM and its obvious generalizations are the first class of non-trivial models in quantum statistical mechanics for which the Green-Kubo formula and the Onsager reciprocity relations have been proven. We would also like to mention related works [AJPP1, AJPP2] where the Green-Kubo formula was established for some exactly solvable quasi-free models. Linear response theory for the quantum Markovian semigroup describing the dynamics of the SFM in the van Hove weak coupling limit was studied by Lebowitz and Spohn in [LeSp] and this work has motivated our program. The Green-Kubo formula for a class of open systems in classical non-equilibrium statistical mechanics has been established in $[\mathrm{RBT}]$.

Acknowledgment. The research of the first author was partly supported by NSERC. A part of this work has been done during the visit of the first author to CPT-CNRS. Y.O. is supported by the Japan Society for the Promotion of Science. This work has been done during the stay of Y.O. to CPT-CNRS, partly supported by the Canon Foundation in Europe and JSPS.

\subsection{The model and the results}

The spin-fermion model describes a two level quantum system $\mathcal{S}$ (spin 1/2) coupled to finitely many, say $M$, free Fermi gas reservoirs $\mathcal{R}_{j}$. This model—a paradigm of open quantum system—has been much studied and we shall be brief in its description. The reader not familiar with the model or with the algebraic formalism of quantum statistical mechanics may consult [JP2] or any of the references [Da, BR1, BR2, LeSp, JP3, FMU, AJPP1] for additional information.

The system $\mathcal{S}$ is described by the Hilbert space $\mathbb{C}^{2}$ and the Hamiltonian $H_{\mathcal{S}}=\sigma_{z}\left(\sigma_{x}, \sigma_{y}, \sigma_{z}\right.$ denote the usual Pauli matrices). Its algebra of observables is the matrix algebra $\mathcal{O}_{\mathcal{S}}=M\left(\mathbb{C}^{2}\right)$ and its dynamics is

$$
\tau_{\mathcal{S}}^{t}(A)=\mathrm{e}^{\mathrm{i} t H_{\mathcal{S}}} A \mathrm{e}^{-\mathrm{i} t H_{\mathcal{S}}} .
$$

A convenient reference state of the system $\mathcal{S}$ is

$$
\omega_{\mathcal{S}}(A)=\frac{1}{2} \operatorname{Tr}(A)
$$

but none of our results depends on this choice. 
The reservoir $\mathcal{R}_{j}$ is a free Fermi gas in thermal equilibrium at inverse temperature $\beta_{j}$ described by the quantum dynamical system $\left(\mathcal{O}_{j}, \tau_{j}, \omega_{j}\right)$, where the algebra of observables $\mathcal{O}_{j}=\operatorname{CAR}\left(\mathfrak{h}_{j}\right)$ is the CAR algebra over a single fermion Hilbert space $\mathfrak{h}_{j}$, the $C^{*}$-dynamics $\tau_{j}^{t}$ is the group of Bogoliubov *-automorphisms generated by a single particle Hamiltonian $h_{j}$, and $\omega_{j}$ is the unique $\left(\tau_{j}, \beta_{j}\right)$-KMS state on $\mathcal{O}_{j}$. We denote by $\delta_{j}$ the generator of $\tau_{j}$. The complete reservoir system $\mathcal{R}=\sum_{j} \mathcal{R}_{j}$ is described by the quantum dynamical system $\left(\mathcal{O}_{\mathcal{R}}, \tau_{\mathcal{R}}, \omega_{\mathcal{R}}\right)$ where

$$
\mathcal{O}_{\mathcal{R}}=\otimes_{j=1}^{M} \mathcal{O}_{j}, \quad \tau_{\mathcal{R}}=\otimes_{j=1}^{M} \tau_{j}, \quad \omega_{\mathcal{R}}=\otimes_{j=1}^{M} \omega_{j} .
$$

Since we are interested in the non-equilibrium statistical mechanics of the SFM, we assume that $M \geq 2$.

Notation. In the sequel, whenever the meaning is clear within the context, we will write $A$ for the operators $A \otimes I$, $I \otimes A$.

In absence of interaction the joint system $\mathcal{S}+\mathcal{R}$ is described by the quantum dynamical system $\left(\mathcal{O}, \tau_{0}, \omega\right)$, where

$$
\mathcal{O}=\mathcal{O}_{\mathcal{S}} \otimes \mathcal{O}_{\mathcal{R}}, \quad \tau_{0}=\tau_{\mathcal{S}} \otimes \tau_{\mathcal{R}}, \quad \omega=\omega_{\mathcal{S}} \otimes \omega_{\mathcal{R}}
$$

We denote by

$$
\delta_{0}=\mathrm{i}\left[H_{\mathcal{S}}, \cdot\right]+\sum_{j=1}^{M} \delta_{j}
$$

the generator of $\tau_{0}$.

The interaction of $\mathcal{S}$ with $\mathcal{R}_{j}$ is described by

$$
V_{j}=\sigma_{x} \otimes \varphi_{j}\left(\alpha_{j}\right)
$$

where $\alpha_{j} \in \mathfrak{h}_{j}$ is a given vector (sometimes called "form-factor"), and

$$
\varphi_{j}\left(\alpha_{j}\right)=\frac{1}{\sqrt{2}}\left(a_{j}\left(\alpha_{j}\right)+a_{j}^{*}\left(\alpha_{j}\right)\right) \in \mathcal{O}_{j},
$$

is the field operator associated to $\alpha_{j}$. The complete interaction between $\mathcal{S}$ and $\mathcal{R}$ is given by $V=\sum_{j=1}^{M} V_{j}$. Let $\lambda \in \mathbb{R}$ be a coupling constant and $\tau_{\lambda}$ the $C^{*}$-dynamics on $\mathcal{O}$ generated by

$$
\delta_{\lambda}=\delta_{0}+\mathrm{i} \lambda[V, \cdot] .
$$

The interacting joint system $\mathcal{S}+\mathcal{R}$ is described by the $C^{*}$-dynamical system $\left(\mathcal{O}, \tau_{\lambda}\right)$ and the reference state $\omega$.

Time-reversal invariance plays an important role in linear response theory. We remark that due to its simple form, the spin-fermion system is automatically time-reversal invariant. Indeed, for all $j$ there exists a complex conjugation $c_{j}$ on $\mathfrak{h}_{j}$ which commutes with $h_{j}$ and satisfies $c_{j} \alpha_{j}=\alpha_{j}$. The map $\Theta_{j}\left(a\left(f_{j}\right)\right)=a\left(c_{j} f_{j}\right)$ uniquely extends to an involutive anti-linear $*$-automorphism of $\mathcal{O}_{j}$ such that $\Theta_{j} \circ \tau_{j}^{t}=\tau_{j}^{-t} \circ \Theta_{j}$. Let $\Theta_{\mathcal{S}}$ be the standard complex conjugation on $\mathcal{O}_{\mathcal{S}}$. Obviously, $\Theta_{\mathcal{S}}\left(\sigma_{z}\right)=\sigma_{z}, \Theta_{\mathcal{S}}\left(\sigma_{x}\right)=\sigma_{x}$, and in particular $\Theta_{\mathcal{S}} \circ \tau_{\mathcal{S}}^{t}=\tau_{\mathcal{S}}^{-t} \circ \Theta_{\mathcal{S}}$. Let $\Theta=\Theta_{\mathcal{S}} \otimes \Theta_{1} \otimes \cdots \Theta_{M}$. Then $\Theta\left(V_{j}\right)=V_{j}$ for all $j$, and $\Theta \circ \tau_{\lambda}^{t}=\tau_{\lambda}^{-t} \circ \Theta$ for all $\lambda \in \mathbb{R}$.

Let $\beta_{\mathrm{eq}}>0$ be a given reference (equilibrium) inverse temperature. Since we are interested in linear response theory, without loss of generality we may restrict the inverse temperatures $\beta_{j}$ of the reservoirs to an interval $\left(\beta_{\mathrm{eq}}-\epsilon, \beta_{\mathrm{eq}}+\epsilon\right)$, where $0<\epsilon<\beta_{\mathrm{eq}}$ is a small number. For our purposes the size of $\epsilon$ is not relevant. We introduce the thermodynamical forces

$$
X_{j}=\beta_{\mathrm{eq}}-\beta_{j},
$$

and set $X=\left(X_{1}, \cdots, X_{M}\right)$. The vector $X$ uniquely describes the initial state of the system (note that the value $X=0$ corresponds to the equilibrium case where all $\beta_{j}$ are the same and equal to $\beta_{\text {eq }}$ ). The restriction $\beta_{j} \in\left(\beta_{\mathrm{eq}}-\epsilon, \beta_{\mathrm{eq}}+\epsilon\right)$ is equivalent to $|X|_{+}<\epsilon$, where $|X|_{+}=\max \left|X_{j}\right|$. We set $\mathbb{I}_{\epsilon}=\left\{\left.X \in \mathbb{R}^{M}|| X\right|_{+}<\epsilon\right\}$, 
$D_{\epsilon}=\left\{\left.X \in \mathbb{C}^{M}|| X\right|_{+}<\epsilon\right\}$. We shall explicitly indicate the dependence of the reference states on $X$ by denoting $\omega_{X_{j}}=\omega_{j}, \omega_{\mathcal{R} X}=\omega_{X_{1}} \otimes \cdots \otimes \omega_{X_{M}}$ and

$$
\omega_{X}^{(0)}=\omega_{\mathcal{S}} \otimes \omega_{\mathcal{R} X}
$$

We denote by $\mathcal{N}_{X}$ the set of all $\omega_{X}^{(0)}$-normal states on $\mathcal{O}$.

We will need several results concerning non-equilibrium thermodynamics of $\mathcal{S}+\mathcal{R}$ established in [JP2]. We first list technical conditions needed for these results.

(A1) $\mathfrak{h}_{j}=L^{2}\left(\mathbb{R}_{+}, \mathrm{d} s ; \mathfrak{H}_{j}\right)$ for some auxiliary Hilbert space $\mathfrak{H}_{j}$ and $h_{j}$ is the operator of multiplication by $s \in \mathbb{R}_{+}$.

Let $I(\delta)=\{z \in \mathbb{C}|| \operatorname{Im} z \mid<\delta\}$ and let $H_{j}^{2}(\delta)$ be the usual Hardy class of analytic functions $f: I(\delta) \rightarrow \mathfrak{H}_{j}$.

(A2) For some $\delta>0, a>\beta_{\text {eq }}$, and all $j$, $\mathrm{e}^{-a s} \alpha_{j}(|s|) \in H_{j}^{2}(\delta)$.

(A3) For all $j,\left\|\alpha_{j}(2)\right\|_{\mathfrak{H}_{j}}>0$.

(A1) and (A2) are regularity assumptions needed for the spectral theory of NESS developed in [JP2]. Assumption (A3) is the "Fermi Golden Rule" condition which ensures that $\mathcal{S}$ is effectively coupled to each reservoir $\mathcal{R}_{j}$.

The following result was proven in [JP2].

Theorem 1.1 Assume that (A1)-(A3) hold. Then, there exist $\Lambda>0, \epsilon>0$ and states $\omega_{\lambda X+}$ on $\mathcal{O}$ such that for $0<|\lambda|<\Lambda, X \in I_{\epsilon}, \eta \in \mathcal{N}_{X}$, and $A \in \mathcal{O}$,

$$
\lim _{t \rightarrow+\infty} \eta\left(\tau_{\lambda}^{t}(A)\right)=\omega_{\lambda X+}(A)
$$

The states $\omega_{\lambda X+}$ are the NESS of the joint system $\mathcal{S}+\mathcal{R}$ and are the central objects of the non-equilibrium statistical mechanics of this system. We remark that $\omega_{\lambda 0+}$ is the unique $\left(\tau_{\lambda}, \beta_{\text {eq }}\right)$-KMS state on $\mathcal{O}$ and in this case Relation (1.2) is the statement of the zeroth law of thermodynamics. We denote $\omega_{\lambda \mathrm{eq}}=\omega_{\lambda 0+}$.

If the thermodynamical forces $X_{j}$ are not all the same, then one expects that the NESS $\omega_{\lambda X+}$ is thermodynamically non-trivial and has strictly positive entropy production. This result was also established in [JP2] (see also [JP3]). The observable describing the heat flux out of $\mathcal{R}_{j}$ is

$$
\Phi_{j}=\lambda \delta_{j}\left(V_{j}\right)=\lambda \sigma_{x} \otimes \varphi_{j}\left(\mathrm{i} h_{j} \alpha_{j}\right) .
$$

The entropy production of the NESS $\omega_{\lambda X+}$ is defined by

$$
\operatorname{Ep}\left(\omega_{\lambda X+}\right)=\sum_{j=1}^{M} X_{j} \omega_{\lambda X+}\left(\Phi_{j}\right) .
$$

If (A1)-(A3) hold and the $X_{j}$ 's are not all the same, then for $\lambda$ non-zero and small enough, $\operatorname{Ep}\left(\omega_{\lambda X+}\right)>0$, see [JP2]. In particular, the NESS $\omega_{\lambda X+}$ carries non-vanishing heat fluxes. We will return to this topic in Subsection 1.2 .

In this paper we study the linear response of $\omega_{\lambda X+}$ to thermodynamical forces $X_{j}$. Our first result is:

Theorem 1.2 Assume that (A1)-(A3) hold. Then there are $\Lambda>0$ and $\epsilon>0$ such that for $0<|\lambda|<\Lambda$ the maps

$$
\mathbb{I}_{\epsilon} \ni X \mapsto \omega_{\lambda X+}\left(\Phi_{j}\right),
$$

extend to analytic functions on $D_{\epsilon}$. 
The kinetic transport coefficients are defined by

$$
L_{\lambda j i}=\left.\partial_{X_{i}} \omega_{\lambda X+}\left(\Phi_{j}\right)\right|_{X=0} .
$$

Our main result is:

Theorem 1.3 Assume that (A1)-(A3) hold. Then there is $\Lambda>0$ such that for $0<|\lambda|<\Lambda$ the following holds:

(1)

$$
L_{\lambda j i}=\frac{1}{2} \int_{-\infty}^{\infty} \omega_{\lambda \mathrm{eq}}\left(\Phi_{j} \tau_{\lambda}^{t}\left(\Phi_{i}\right)\right) \mathrm{d} t
$$

$$
L_{\lambda j i}=L_{\lambda i j}
$$

Remark 1. The relations (1.4) are the Green-Kubo formulas for heat fluxes. The relations (1.5) are the Onsager reciprocity relations.

Remark 2. The Onsager reciprocity relations are an immediate consequence of (1.4). Indeed, Theorem 1.1 yields that for $A, B \in \mathcal{O}$,

$$
\lim _{|t| \rightarrow \infty} \omega_{\lambda \text { eq }}\left(A \tau_{\lambda}^{t}(B)\right)=\omega_{\lambda \text { eq }}(A) \omega_{\lambda \text { eq }}(B) .
$$

This fact implies that

$$
\lim _{T \rightarrow \infty} \int_{-T}^{T} \omega_{\lambda \mathrm{eq}}\left(\left[\Phi_{j}, \tau_{\lambda}^{t}\left(\Phi_{i}\right)\right]\right) \mathrm{d} t=0
$$

see Theorem 5.14.12 in [BR2]. Since $\omega_{\lambda \text { eq }}$ is obviously $\tau_{\lambda}$-invariant, (1.4) implies (1.5).

Remark 3. In general, in the Green-Kubo formula $\int_{-\infty}^{\infty}$ is interpreted as $\lim _{T \rightarrow \infty} \int_{-T}^{T}$. However, since $\omega_{\lambda \text { eq }}\left(\Phi_{j}\right)=$ 0 for all $j$, by the result of [JP2]

$$
\left|\omega_{\lambda \mathrm{eq}}\left(\Phi_{j} \tau_{\lambda}^{t}\left(\Phi_{i}\right)\right)\right|=O\left(\mathrm{e}^{-A|t|}\right)
$$

for some $A>0$, and the integral in (1.4) is absolutely convergent.

Our final result is:

Theorem 1.4 Assume that (A1)-(A3) hold. Then there is $\Lambda>0$ such that the functions $\lambda \mapsto L_{\lambda j i}$ are analytic for $|\lambda|<\Lambda$ and have power expansions

$$
L_{\lambda j i}=\sum_{k=2}^{\infty} \lambda^{k} L_{j i}^{(k)}
$$

Moreover, for $j \neq i$,

$$
L_{j i}^{(2)}=-\frac{\pi}{\left(\cosh \beta_{\text {eq }}\right)^{2}} \frac{\left\|\alpha_{i}(2)\right\|_{\mathfrak{H}_{i}}^{2}\left\|\alpha_{j}(2)\right\|_{\mathfrak{H}_{j}}^{2}}{\sum_{k}\left\|\alpha_{k}(2)\right\|_{\mathfrak{H}_{k}}^{2}}
$$

and $L_{i i}^{(2)}=-\sum_{j \neq i} L_{j i}^{(2)}$.

Remark. Starting with formula (1.4), this theorem can be proven by an explicit computation based on the spectral theory of the standard Liouvillean [JP2]. Our proof in Section 4 is somewhat indirect and emphasizes the important connection between $L_{j i}^{(2)}$ and the weak coupling Green-Kubo formula established in [LeSp]. This connection is discussed in more detail in Subsection 1.3 


\subsection{Thermodynamics of the SFM revisited}

Some technical results established in this paper could be used to improve existing results concerning the thermodynamics of the SFM. In this subsection we do not assume that $\epsilon$ is small and $\beta_{\text {eq }}$ does not play any particular role. For this reason, in this subsection we replace the subscripts $X$ by $\vec{\beta}=\left(\beta_{1}, \cdots, \beta_{M}\right)$. Hence, $\omega_{\beta_{j}}=\omega_{j}$ is the initial state of the reservoir $\mathcal{R}_{j}, \omega_{\mathcal{R} \vec{\beta}}=\omega_{\beta_{1}} \otimes \cdots \otimes \omega_{\beta_{M}}, \omega_{\vec{\beta}}=\omega_{\mathcal{S}} \otimes \omega_{\mathcal{R} \vec{\beta}}$ is the reference state of the joint system, $\mathcal{N}_{\vec{\beta}}$ is the set of all $\omega_{\vec{\beta}}$-normal states on $\mathcal{O}$, etc. For $0<\gamma_{1}<\gamma_{2}$ we denote $\mathbb{I}_{\gamma_{1} \gamma_{2}}=\left[\gamma_{1}, \gamma_{2}\right]^{M} \subset \mathbb{R}^{M}$. In this subsection we will always assume the constant $a$ in Assumption (A2) satisfies $a>\gamma_{2}$.

The following results hold:

Theorem 1.5 Let $0<\gamma_{1}<\gamma_{2}$ be given and assume that (A1)-(A3) hold. Then there exist $\Lambda>0$ and states $\omega_{\lambda \vec{\beta}+}$ on $\mathcal{O}$ such that:

(1) For all $0<|\lambda|<\Lambda, \vec{\beta} \in \mathbb{I}_{\gamma_{1} \gamma_{2}}, \eta \in \mathcal{N}_{\vec{\beta}}$, and $A \in \mathcal{O}$,

$$
\lim _{t \rightarrow+\infty} \eta\left(\tau_{\lambda}^{t}(A)\right)=\omega_{\lambda \vec{\beta}+}(A) .
$$

(2) The limit (1.8) is exponentially fast in the following sense: There exist $\rho_{\lambda \vec{\beta}}>0$, a norm dense set of states $\mathcal{N}_{0 \vec{\beta}} \subset \mathcal{N}_{\vec{\beta}}$, and a norm-dense $*$-subalgebra $\mathcal{O}_{0} \subset \mathcal{O}$ such that for $\eta \in \mathcal{N}_{0 \vec{\beta}}, A \in \mathcal{O}_{0}$, and $t>0$,

$$
\left|\eta\left(\tau_{\lambda}^{t}(A)\right)-\omega_{\lambda \vec{\beta}+}(A)\right| \leq C_{A, \eta, \lambda} \mathrm{e}^{-\rho_{\lambda \vec{\beta}} t} .
$$

Moreover, $\omega_{\vec{\beta}} \in \mathcal{N}_{0 \vec{\beta}}$, $\Phi_{j} \in \mathcal{O}_{0}$, and

$$
\rho_{\lambda \vec{\beta}}=\frac{\pi}{2}\left(\sum_{j}\left\|\alpha_{j}(2)\right\|_{\mathfrak{H}_{j}}^{2}\right) \lambda^{2}+O\left(\lambda^{4}\right),
$$

where the reminder is uniform in $\vec{\beta} \in \mathbb{I}_{\gamma_{1} \gamma_{2}}$.

(3) There exists a neighborhood $O_{\gamma_{1} \gamma_{2}}$ of $\mathbb{I}_{\gamma_{1} \gamma_{2}}$ in $\mathbb{C}^{M}$ such that for all $A \in \mathcal{O}_{0}$ the functions

$$
(\lambda, \vec{\beta}) \mapsto \omega_{\lambda \vec{\beta}+}(A),
$$

extend to analytic functions on $\{\lambda|| \lambda \mid<\Lambda\} \times O_{\gamma_{1} \gamma_{2}}$.

Remark. Parts (1) and (2) are proven in [JP2] and are stated here for reference purposes. The new result is (3) - in [JP2] the analyticity of the functions (1.11) was discussed only w.r.t. $\lambda$.

We denote by $\hat{\mathbb{I}}_{\gamma_{1} \gamma_{2}}$ the "off-diagonal" part of $\mathbb{I}_{\gamma_{1} \gamma_{2}}$, i.e.,

$$
\hat{\mathbb{I}}_{\gamma_{1} \gamma_{2}}=\mathbb{I}_{\gamma_{1} \gamma_{2}} \backslash\left\{\vec{\beta} \mid \beta_{1}=\cdots=\beta_{M}\right\} .
$$

Theorem 1.6 Let $0<\gamma_{1}<\gamma_{2}$ be given and assume that (A1)-(A3) hold. Then there exists $\Lambda>0$ such that for $0<|\lambda|<\Lambda$ and $\vec{\beta} \in \hat{\mathbb{I}}_{\gamma_{1} \gamma_{2}}$ the following holds:

(1) $\operatorname{Ep}\left(\omega_{\lambda \vec{\beta}+}\right)>0$.

(2) There are no $\tau_{\lambda}$-invariant states in $\mathcal{N}_{\vec{\beta}}$.

Remark 1. Statements (1) and (2) are equivalent. Indeed, the exponentially fast approach to NESS (Part (2) of Theorem 1.5) and Theorem 1.1 in [JP2] yield that (2) implies (1). On the other hand, if $\eta$ is a normal $\tau_{\lambda^{-}}$ invariant state in $\mathcal{N}_{\vec{\beta}}$, then, by Part (1) of Theorem 1.5, $\eta=\omega_{\lambda \vec{\beta}+}$. This fact and Theorem 1.3 in [JP5] yield that $\operatorname{Ep}\left(\omega_{\lambda \vec{\beta}+}\right)=0$, and so (2) implies (1).

Remark 2. Theorem 1.6 was proven in [JP2] under the additional assumption that for some $\delta>0$,

$$
\sum_{i, j}\left|\beta_{i}-\beta_{j}\right|>\delta \text {. }
$$

The constant $\Lambda$ was dependent on $\delta$.

Remark 3. A result related to Part (2) of Theorem 1.6 was recently established in [MMS].

The proofs of Theorems 1.5 and 1.6 are given in Section 5. 


\subsection{Some generalizations}

All our results easily extend to more general models where $\mathcal{S}$ is a $N$-level atom described by the Hilbert space $\mathbb{C}^{N}$ and the Hamiltonian $H_{\mathcal{S}}$. Each $V_{j}$ is a finite sum of terms of the form

$$
Q_{j, k} \otimes \varphi_{j}\left(\alpha_{j, k, 1}\right) \cdots \varphi_{j}\left(\alpha_{j, k, n_{j, k}}\right)+\text { h.c., }
$$

where $n_{j, k} \geq 1, Q_{j, k} \in \mathcal{O}_{\mathcal{S}}=M\left(\mathbb{C}^{N}\right)$ and $\alpha_{j, k, n} \in \mathfrak{h}_{j}$ satisfy:

(A0) If $k \neq l$ or $n \neq m$, then $\left(\alpha_{j, k, n}, \mathrm{e}^{\mathrm{i} t h_{j}} \alpha_{j, l, m}\right)=0$ for all $t \in \mathbb{R}$.

We shall call this model the general spin-fermion model (abbreviated GSFM). The GSFM may not be timereversal invariant. Assume that (A1) holds. Let $c_{j}$ be a distinguished complex conjugation on $\mathfrak{h}_{j}$ and

$$
\tilde{\alpha}_{j, k, n}(s)= \begin{cases}\alpha_{j, k, n}(s) & \text { if } s \geq 0 \\ \left(c_{j} \alpha_{j, k, n}\right)(|s|) & \text { if } s<0\end{cases}
$$

Assumption (A2) is replaced with

(A4) For some $\delta>0, a>\beta_{\text {eq }}$, and all $j, k, n, \mathrm{e}^{-a s} \tilde{\alpha}_{j, k, n}(s) \in H_{j}^{2}(\delta)$.

The general "Fermi Golden Rule" non-degeneracy condition is formulated as follows. Assumptions (A0), (A1) and (A4) ensure that for all $X$ there exists a linear map $K_{X}: \mathcal{O}_{\mathcal{S}} \rightarrow \mathcal{O}_{\mathcal{S}}$ such that for all $A, B \in \mathcal{O}_{\mathcal{S}}$,

$$
\lim _{t \rightarrow+\infty} \omega_{X}^{(0)}\left(A \tau_{0}^{-t / \lambda^{2}} \circ \tau_{\lambda}^{t / \lambda^{2}}(B)\right)=\frac{1}{N} \operatorname{Tr}\left(A \mathrm{e}^{t K_{X}}(B)\right) .
$$

As usual, we write $K_{0}=K_{\text {eq }}$. This relation (the quantum Markovian semigroup approximation of the dynamics of an open quantum system in the van Hove weak coupling limit) is a celebrated result of Davies [Da] who has proven it under very general technical conditions (see also [De, JP2, JP3]). The result of Davies was the starting point of numerous studies of thermodynamics of open quantum systems in weak coupling limit (see [LeSp, AJPP1] for references and additional information). We will return to this point at the end of this subsection.

We recall that he generator $K_{X}$ has the form

$$
K_{X}=\sum_{j=1}^{M} K_{X_{j}},
$$

where $K_{X_{j}}$ is the generator obtained by considering the weak coupling limit of the system $\mathcal{S}+\mathcal{R}_{j}$ w.r.t. the initial state $\omega_{\mathcal{S}} \otimes \omega_{X_{j}}$. By construction, the spectrum of $K_{X_{j}}$ is contained in $\{z \mid \operatorname{Re} z \leq 0\}$ and $0 \in \sigma\left(K_{X_{j}}\right)$. Assumption (A3) is replaced with

(A5) For all $j$ and $\left|X_{j}\right|<\epsilon, \sigma\left(K_{X_{j}}\right) \cap i \mathbb{R}=\{0\}$ and 0 is a simple eigenvalue of $K_{X_{j}}$.

In the literature one can find various algebraic characterizations of (A5) (see [Sp, De] for references and additional information).

If Assumptions (A1), (A4) and (A5) hold, then Theorem 1.1 holds for the GSFM. The heat fluxes are again defined by $\Phi_{j}=\lambda \delta_{j}\left(V_{j}\right)$, and if not all $X_{j}$ 's are the same, the entropy production of $\omega_{\lambda X+}$ is strictly positive for small $\lambda$ (see [JP2, JP3]).

Our next assumption concerns time-reversal invariance.

(A6) The complex conjugations $c_{j}$ commute with $h_{j}$ and satisfy $c_{j} \alpha_{j, k, n}=\alpha_{j, k, n}$ for all $j, k, n$. Moreover, the matrices $H_{\mathcal{S}}$ and $Q_{j, k}$ are real w.r.t. the usual complex conjugation on $M\left(\mathbb{C}^{N}\right)$. 
This assumption ensures that there exists an involutive, anti-linear $*$-automorphism (time-reversal) $\Theta$ of $\mathcal{O}$ such that for all $j, \Theta\left(V_{j}\right)=V_{j}, \Theta \circ \tau_{j}^{t}=\tau_{j}^{-t} \circ \Theta$, and $\Theta \circ \tau_{\mathcal{S}}^{t}=\tau_{\mathcal{S}}^{-t} \circ \Theta$. In particular, $\Theta \circ \tau_{\lambda}^{t}=\tau_{\lambda}^{-t} \circ \Theta$ for all $\lambda \in \mathbb{R}$.

Our main result concerning the GSFM is:

Theorem 1.7 Assume that (AO), (A1), (A4) and (A5) hold. Then there are $\Lambda>0$ and $\epsilon>0$ such that for $0<|\lambda|<\Lambda$ the following holds.

(1) The maps

$$
\mathbb{I}_{\epsilon} \ni X \mapsto \omega_{\lambda X+}\left(\Phi_{j}\right)
$$

extend to analytic functions on $D_{\epsilon}$.

(2) Let $L_{\lambda j i}$ be given by (1.3). Then for all $j, i$,

$$
L_{\lambda j i}=\frac{1}{\beta_{\text {eq }}} \int_{0}^{\infty} \mathrm{d} t \int_{0}^{\beta_{\text {eq }}} \mathrm{d} u \omega_{\lambda \text { eq }}\left(\tau_{\lambda}^{t}\left(\Phi_{j}\right) \tau_{\lambda}^{\mathrm{i} u}\left(\Phi_{i}\right)\right) .
$$

(3) Assume in addition that (A6) holds. Then for all $j, i$,

$$
L_{\lambda j i}=\frac{1}{2} \int_{-\infty}^{\infty} \omega_{\lambda \mathrm{eq}}\left(\Phi_{j} \tau_{\lambda}^{t}\left(\Phi_{i}\right)\right) \mathrm{d} t
$$

and

$$
L_{\lambda j i}=L_{\lambda i j}
$$

Remark. The relation (1.13) is the Green-Kubo formula without time reversal assumption. For additional discussion of this point we refer the reader to [JOP2].

Before discussing the generalization of Theorem 1.4 we recall a few basic definitions and results of the weak coupling (sometimes also called Fermi Golden Rule or FGR) thermodynamics of open quantum systems. Assumption (A5) ensures that there exists a density matrix $\omega_{\mathcal{S} X}$ on $\mathcal{H}_{\mathcal{S}}$ such that for any initial density matrix $\rho$ on $\mathcal{H}_{\mathcal{S}}$ and $A \in \mathcal{O}_{\mathcal{S}}$,

$$
\lim _{t \rightarrow+\infty} \operatorname{Tr}\left(\rho \mathrm{e}^{t K_{X}}(A)\right)=\operatorname{Tr}\left(\omega_{\mathcal{S} X+} A\right) \equiv \omega_{\mathcal{S} X+}(A)
$$

The density matrix $\omega_{\mathcal{S} X+}$ is the weak coupling NESS of the open quantum system $\mathcal{S}+\sum_{j} \mathcal{R}_{j}$. Clearly,

$$
\omega_{\mathcal{S} 0+}=\mathrm{e}^{-\beta_{\mathrm{eq}} H_{\mathcal{S}}} / \operatorname{Tr}\left(\mathrm{e}^{-\beta_{\mathrm{eq}} H_{\mathcal{S}}}\right),
$$

and we will write $\omega_{\mathcal{S} 0+}=\omega_{\mathcal{S} \text { eq }}$. Weak coupling heat flux observables are defined by $\bar{\Phi}_{j X}=K_{X_{j}}\left(H_{\mathcal{S}}\right)$ and we denote $\bar{\Phi}_{j \text { eq }}=\bar{\Phi}_{j 0}$. The weak coupling entropy production is

$$
\overline{\mathrm{Ep}}=\sum_{j=1}^{M} X_{j} \omega_{\mathcal{S} X+}\left(\bar{\Phi}_{j X}\right)
$$

One always has $\overline{\mathrm{Ep}} \geq 0$. Lebowitz and Spohn [LeSp] have shown that if (A4) holds then $\overline{\mathrm{Ep}}>0$ whenever $X_{j}$ are not all equal. In the same paper they have also proven the Green-Kubo formula for weak coupling heat fluxes: If (A5) holds, then the functions $X \mapsto \omega_{\mathcal{S} X+}\left(\bar{\Phi}_{j X}\right)$ are differentiable at $X=0$ and

$$
\left.\bar{L}_{j i} \equiv \partial_{X_{i}} \omega_{\mathcal{S} X+}\left(\Phi_{j X}\right)\right|_{X=0}=\int_{0}^{\infty} \omega_{\mathcal{S e q}}\left(\mathrm{e}^{t K_{\mathrm{eq}}}\left(\bar{\Phi}_{j \mathrm{eq}}\right) \bar{\Phi}_{i \mathrm{eq}}\right) \mathrm{d} t
$$

These results are very robust and can be derived under very mild technical conditions. If in addition (A6) holds, then $\bar{L}_{j i}=\bar{L}_{i j}$, that is, the weak coupling Onsager reciprocity relations hold. 
One naturally expects that the weak coupling thermodynamics is the first non-trivial contribution (in $\lambda$ ) to the microscopic thermodynamics. Indeed, it was proven in [JP2, JP3] that if (A0), (A4) and (A5) hold, then for $A \in \mathcal{O}_{\mathcal{S}}$ and $\lambda$ small enough,

$$
\begin{aligned}
\omega_{\lambda X+}(A) & =\omega_{\mathcal{S} X+}(A)+O(\lambda), \\
\omega_{\lambda X+}\left(\Phi_{j}\right) & =\lambda^{2} \omega_{\mathcal{S} X+}\left(\bar{\Phi}_{j X}\right)+O\left(\lambda^{3}\right), \\
\operatorname{Ep}\left(\omega_{\lambda X+}\right) & =\lambda^{2} \overline{\operatorname{Ep}}+O\left(\lambda^{3}\right) .
\end{aligned}
$$

In the next theorem we relate $L_{\lambda j i}$ and $\bar{L}_{j i}$ and complete the link between the microscopic and the weak coupling thermodynamics for this class of models.

Theorem 1.8 Assume that (A0), (A1), (A4) and (A5) hold. Then there is $\Lambda>0$ such that the functions $\lambda \mapsto L_{\lambda j i}$ are analytic for $|\lambda|<\Lambda$ and have power expansions

$$
L_{\lambda j i}=\sum_{k=2}^{\infty} \lambda^{k} L_{j i}^{(k)} .
$$

Moreover,

$$
L_{j i}^{(2)}=\bar{L}_{j i}
$$

Remark 1. Relation (1.16) yields

$$
\lim _{\lambda \rightarrow 0} \lambda^{-2} \frac{1}{\beta_{\text {eq }}} \int_{0}^{\infty} \mathrm{d} t \int_{0}^{\beta_{\text {eq }}} \mathrm{d} u \omega_{\lambda \text { eq }}\left(\tau_{\lambda}^{t}\left(\Phi_{j}\right) \tau_{\lambda}^{\mathrm{i} u}\left(\Phi_{i}\right)\right) \mathrm{d} t=\int_{0}^{\infty} \omega_{\mathcal{S} \text { eq }}\left(\mathrm{e}^{t K_{\text {eq }}}\left(\bar{\Phi}_{j \mathrm{eq}}\right) \bar{\Phi}_{i \mathrm{eq}}\right) \mathrm{d} t,
$$

and if in addition (A6) holds

$$
\lim _{\lambda \rightarrow 0} \lambda^{-2} \int_{-\infty}^{\infty} \omega_{\lambda \mathrm{eq}}\left(\tau_{\lambda}^{t}\left(\Phi_{j}\right) \Phi_{i}\right) \mathrm{d} t=\int_{0}^{\infty} \omega_{\mathcal{S e q}}\left(\mathrm{e}^{t K_{\mathrm{eq}}}\left(\bar{\Phi}_{j \mathrm{eq}}\right) \bar{\Phi}_{i \mathrm{eq}}\right) \mathrm{d} t
$$

i.e. the rescaled microscopic flux-flux correlation functions converge to the corresponding weak coupling correlation functions.

Remark 2. The relation between the microscopic and the weak coupling thermodynamics is discussed in detail in the lecture notes [AJPP1] in the context of an exactly solvable quasi-free model.

The proofs of Theorems 1.7 and 1.8 are only notationally different from the proofs of Theorems 1.3 and 1.4 and the details can be found in the forthcoming review article [JP4].

Theorems 1.5 and 1.6 also hold for the GSFM under the Assumptions (A0), (A1), (A4) with $a>\gamma_{2}$, and (A5) for all $\vec{\beta} \in \mathbb{I}_{\gamma_{1} \gamma_{2}}$. The only parts that need to be modified are Relations (1.9) and (1.10). In general, the constant $C_{A, \eta, \lambda}$ is replaced by a polynomial in $t$. The leading term in the expansion (1.10) is equal to the absolute value of the real part of the non-zero eigenvalue of $K_{\vec{\beta}}$ closest to i $\mathbb{R}$ and in general depends on $\vec{\beta}$. For additional discussion of these points we refer the reader to [JP4].

\section{Strategy of the proof}

The proofs of Theorems 1.2 and 1.3 are based on two ingredients: the abstract derivation of the Green-Kubo formula in [JOP1, JOP2] and the detailed information about the NESS of the SFM obtained in [JP2]. In this section we outline how to combine these ingredients and extract a specific technical result needed to complete the 
proofs. This technical result will be established in the next section. Throughout this subsection we assume that (A1)-(A3) hold.

Consider the $C^{*}$-dynamics $\sigma_{X}^{(0)}$ on $\mathcal{O}$ generated by

$$
\delta_{X}^{(0)}=\sum_{j}\left(1-X_{j} / \beta_{\mathrm{eq}}\right) \delta_{j}
$$

The state $\omega_{X}^{(0)}$ is the unique $\left(\sigma_{X}^{(0)}, \beta_{\text {eq }}\right)$-KMS state on $\mathcal{O}$. Let $\sigma_{\lambda X}$ be the $C^{*}$-dynamics on $\mathcal{O}$ generated by

$$
\delta_{\lambda X}=\delta_{X}^{(0)}+\mathrm{i}\left[H_{\mathcal{S}}+\lambda V, \cdot\right]=\delta_{\lambda}-\sum_{j} \frac{X_{j}}{\beta_{\mathrm{eq}}} \delta_{j}
$$

The Araki perturbation theory [Ar, BR2, DJP] yields that there exists a unique $\left(\sigma_{\lambda X}, \beta_{\text {eq }}\right)$-KMS state on $\mathcal{O}$. We denote this state by $\omega_{\lambda X}$. The states $\omega_{X}^{(0)}$ and $\omega_{\lambda X}$ are mutually normal and in particular $\omega_{\lambda X} \in \mathcal{N}_{X}$.

Lemmas 3.5 in [JOP1] and 4.6 in [JOP2] yield that for all $\lambda$ and $t$ the function

$$
X \mapsto \omega_{\lambda X}\left(\tau_{\lambda}^{t}\left(\Phi_{j}\right)\right),
$$

is differentiable at $X=0$. Theorem 2.3 in [JOP1] specialized to the SFM yields:

Theorem 2.1 Let $\Lambda$ be as in Theorem 1.1 and let $0<|\lambda|<\Lambda$ be given. Suppose also that:

(a) The function $X \mapsto \omega_{\lambda X+}\left(\Phi_{j}\right)$ is differentiable at $X=0$.

(b) The limit and the derivative in the expression

$$
\left.\lim _{t \rightarrow+\infty} \partial_{X_{i}} \omega_{\lambda X}\left(\tau_{\lambda}^{t}\left(\Phi_{j}\right)\right)\right|_{X=0}
$$

can be interchanged.

Then,

$$
\partial_{X_{i}} \omega_{\lambda X+}\left(\Phi_{j}\right)=\frac{1}{2} \int_{-\infty}^{\infty} \omega_{\lambda \mathrm{eq}}\left(\Phi_{j} \tau_{\lambda}^{t}\left(\Phi_{i}\right)\right) \mathrm{d} t
$$

By this result, the proof of Theorem 1.3 reduces to the verification of (a) and (b) in Theorem 2.1. We remark that (a) and (b) have been assumed as axioms in the abstract derivation of [JOP1, JOP2]. In study of concrete models they are the central technical points that need to be verified. This brings us to the second point of the proof, namely the dynamical properties of the SFM established in [JP2]. Using the results of [JP2], in the next section we will prove:

Theorem 2.2 There exist $\Lambda>0$ and $\epsilon>0$ such that for $0<|\lambda|<\Lambda$ and $t \geq 0$ the functions $X \mapsto \omega_{\lambda X}\left(\tau_{\lambda}^{t}\left(\Phi_{j}\right)\right)$ have an analytic extension to $D_{\epsilon}$. Moreover,

$$
\sup _{X \in D_{\epsilon}, t \geq 0}\left|\omega_{\lambda X}\left(\tau_{\lambda}^{t}\left(\Phi_{j}\right)\right)\right|<\infty .
$$

This theorem is our key technical result. Theorems 1.1 and 2.2 yield:

Theorem 2.3 Let $\Lambda$ and $\epsilon$ be as in Theorem 2.2. Then for all $X \in D_{\epsilon}$ and $0<|\lambda|<\Lambda$ the limits

$$
h_{\lambda j}(X)=\lim _{t \rightarrow+\infty} \omega_{\lambda X}\left(\tau_{\lambda}^{t}\left(\Phi_{j}\right)\right),
$$

exist. The limiting functions $X \mapsto h_{\lambda j}(X)$ are analytic on $D_{\epsilon}$. Moreover, as $t \rightarrow+\infty$, all derivatives of the functions $X \mapsto \omega_{\lambda X}\left(\tau_{\lambda}^{t}\left(\Phi_{j}\right)\right)$ converge uniformly on compact subsets of $D_{\epsilon}$ to the corresponding derivatives of $h_{\lambda j}(X)$. 
Proof. This result follows from the multivariable Vitali theorem. We sketch the proof for the reader convenience. Set $h_{t}(X)=\omega_{\lambda X}\left(\tau_{\lambda}^{t}\left(\Phi_{j}\right)\right)$. For $0<\rho<\epsilon$ we denote

$$
\mathbb{T}_{\rho}=\left\{X \in \mathbb{C}^{M}|| X_{j} \mid=\rho \text { for all } j\right\} .
$$

The Cauchy integral formula for polydisk yields that for $X \in D_{\rho}$,

$$
h_{t}(X)=\frac{1}{(2 \pi \mathrm{i})^{M}} \int_{\mathbb{T}_{\rho}} \frac{h_{t}\left(\xi_{1}, \cdots, \xi_{M}\right)}{\left(\xi_{1}-X_{1}\right) \cdots\left(\xi_{M}-X_{M}\right)} \mathrm{d} \xi_{1} \cdots \mathrm{d} \xi_{M} .
$$

It follows that the family of functions $\left\{h_{t}\right\}_{t \geq 0}$ is equicontinuous on $D_{\rho^{\prime}}$ for any $\rho^{\prime}<\rho$. Hence, by the ArzelaAscoli theorem, for any $\rho^{\prime}<\rho$ the set $\left\{h_{t}\right\}_{t \geq 0}$ is precompact in the Banach space $C\left(\bar{D}_{\rho^{\prime}}\right)$ of all continuous functions on $\bar{D}_{\rho^{\prime}}$ equipped with the sup norm. The Cauchy integral formula (2.17), where now $X \in D_{\rho^{\prime}}$ and the integral is over $\mathbb{T}_{\rho^{\prime}}$, yields that any limit in $C\left(\bar{D}_{\rho^{\prime}}\right)$ of the net $\left\{h_{t}\right\}$ as $t \rightarrow \infty$ is an analytic function in $D_{\rho^{\prime}}$. By Theorem 1.1, any two limit functions coincide for $X$ real, and hence they are identical. This yields the first part of the theorem. The convergence of partial derivatives of $h_{t}(X)$ is an immediate consequence of the Cauchy integral formula.

Theorem 2.3 yields Theorem 1.2- the functions $h_{\lambda j}(X)$ are the analytic extensions of $\omega_{\lambda X+}\left(\Phi_{j}\right)$ to $D_{\epsilon}$. In particular, assumption (a) of Theorem 2.1 holds. Similarly, the second part of Theorem 2.3 implies that assumption (b) of Theorem 2.1 holds and Theorem 1.3 follows. Hence, to complete the proofs of Theorems 1.2 and 1.3 it remains to establish Theorem 2.2.

\section{Proof of Theorem 2.2}

The proof of Theorem 2.2 is based on techniques and estimates of [JP1, JP2]. We recall the ingredients we need. Throughout this section we assume that (A1)-(A3) hold. $\mathcal{B}(\mathfrak{H})$ denotes the $C^{*}$-algebra of all bounded operators on the Hilbert space $\mathfrak{H}$.

The GNS-representation of the algebra $\mathcal{O}$ associated to the product state $\omega_{X}^{(0)}$ can be explicitly computed [AW]. We will describe it in the glued form of [JP2]. Denote by $e_{ \pm}$the eigenvectors of $\sigma_{z}$ associated to the eigenvalues \pm 1 . Set $\mathcal{H}_{\mathcal{S}}=\mathbb{C}^{2} \otimes \mathbb{C}^{2}$ and define a unit vector in $\mathcal{H}_{\mathcal{S}}$ by

$$
\Omega_{\mathcal{S}}=\frac{1}{\sqrt{2}}\left(e_{-} \otimes e_{-}+e_{+} \otimes e_{+}\right)
$$

Let $\pi_{\mathcal{S}}: \mathcal{O}_{\mathcal{S}} \rightarrow \mathcal{B}\left(\mathcal{H}_{\mathcal{S}}\right)$ be given by

$$
\pi_{\mathcal{S}}(A)=A \otimes I .
$$

The triple $\left(\mathcal{H}_{\mathcal{S}}, \pi_{\mathcal{S}}, \Omega_{\mathcal{S}}\right)$ is the GNS representation of $\mathcal{O}_{\mathcal{S}}$ associated to $\omega_{\mathcal{S}}$. We set

$$
\mathcal{L}_{\mathcal{S}}=H_{\mathcal{S}} \otimes I-I \otimes H_{\mathcal{S}} .
$$

Let $\mathcal{F}_{j}$ be the anti-symmetric Fock space over $\tilde{\mathfrak{h}}_{j}=L^{2}\left(\mathbb{R}, \mathrm{d} s ; \mathfrak{H}_{j}\right)$ and $\Omega_{j}$ the vacuum vector in $\mathcal{F}_{j}$. We denote by $\tilde{a}_{j}, \tilde{a}_{j}^{*}$ the annihilation and creation operators and by $N_{j}$ the number operator on $\mathcal{F}_{j}$. Let $\mathcal{L}_{j}=\mathrm{d} \Gamma(s)$ be the second quantization of the operator of multiplication by $s$ on $\tilde{\mathfrak{h}}_{j}$. To any $f_{j} \in \mathfrak{h}_{j}$ we associate $\tilde{f}_{j} \in \tilde{\mathfrak{h}}_{j}$ by

$$
\tilde{f}_{j}(s)= \begin{cases}f_{j}(s) & \text { if } s \geq 0 \\ \left(c_{j} f_{j}\right)(|s|) & \text { if } s<0 .\end{cases}
$$

For $X \in \mathbb{R}^{M}$ we set

$$
\tilde{f}_{j X}(s)=\left(\mathrm{e}^{\left(X_{j}-\beta_{\mathrm{eq}}\right) s}+1\right)^{-1 / 2} \tilde{f}_{j}(s) .
$$


Finally, we define a map $\pi_{j X}: \mathcal{O}_{j} \rightarrow \mathcal{B}\left(\mathcal{F}_{j}\right)$ by

$$
\pi_{j X}\left(\varphi_{j}\left(f_{j}\right)\right)=\tilde{\varphi}_{j}\left(\tilde{f}_{j X}\right)=\frac{1}{\sqrt{2}}\left(\tilde{a}_{j}\left(\tilde{f}_{j X}\right)+\tilde{a}_{j}^{*}\left(\tilde{f}_{j X}\right)\right) .
$$

The map $\pi_{j X}$ uniquely extends to a representation of $\mathcal{O}_{j}$ on the Hilbert space $\mathcal{F}_{j}$.

We set

$$
\mathcal{H}_{\mathcal{R}}=\otimes_{j=1}^{M} \mathcal{F}_{j}, \quad \pi_{\mathcal{R} X}=\otimes_{j=1}^{M} \pi_{j X}, \quad \Omega_{\mathcal{R}}=\otimes_{j=1}^{M} \Omega_{j}
$$

The triple $\left(\mathcal{H}_{\mathcal{R}}, \pi_{\mathcal{R} X}, \Omega_{\mathcal{R}}\right)$ is the GNS representation of the algebra $\mathcal{O}_{\mathcal{R}}$ associated to the state $\omega_{\mathcal{R} X}$. Let

$$
\mathcal{H}=\mathcal{H}_{\mathcal{S}} \otimes \mathcal{H}_{\mathcal{R}}, \quad \pi_{X}=\pi_{\mathcal{S}} \otimes \pi_{\mathcal{R} X}, \quad \Omega=\Omega_{\mathcal{S}} \otimes \Omega_{\mathcal{R}}
$$

The triple $\left(\mathcal{H}, \pi_{X}, \Omega\right)$ is the GNS-representation of the algebra $\mathcal{O}$ associated to the state $\omega_{X}^{(0)}$. Note that $\mathcal{H}$ and $\Omega$ do not depend on $X$.

The spectral theory of NESS is based on a particular non-selfadjoint operator acting on $\mathcal{H}$, the adjoint of the so-called $C$-Liouvillean. This operator is defined as follows. Let $\mathcal{L}_{\mathcal{R}}=\sum_{j} \mathcal{L}_{j}$ and

$$
\mathcal{L}_{0}=\mathcal{L}_{\mathcal{S}}+\mathcal{L}_{\mathcal{R}}
$$

Let

$$
\begin{aligned}
V_{j X} & =\pi_{X}\left(V_{j}\right)=\sigma_{x} \otimes I \otimes \tilde{\varphi}_{j}\left(\tilde{\alpha}_{j X}\right), \\
W_{j X} & =I \otimes \sigma_{x} \otimes \frac{1}{\sqrt{2}}(-I)^{N_{j}}\left(\tilde{a}_{j}^{*}\left(\mathrm{e}^{\left(X_{j}-\beta_{\mathrm{eq}}\right) s} \tilde{\alpha}_{j X}\right)-\tilde{a}_{j}\left(\tilde{\alpha}_{j X}\right)\right),
\end{aligned}
$$

and

$$
V_{X}=\sum_{j} V_{j X}, \quad W_{X}=\sum_{j} W_{j X}
$$

The adjoint of the $C$-Liouvillean associated to the triple $\left(\mathcal{O}, \tau_{\lambda}, \omega_{X}^{(0)}\right)$ is

$$
\mathcal{L}_{\lambda X}=\mathcal{L}_{0}+\lambda\left(V_{X}+W_{X}\right)
$$

This operator is closed on $\operatorname{Dom}\left(\mathcal{L}_{0}\right)$ and generates a quasi-bounded strongly continuous group $\mathrm{e}^{\mathrm{i} t \mathcal{L}_{\lambda X}}$ on $\mathcal{H}$. The operator $\mathcal{L}_{\lambda X}$ is characterized by the following two properties:

(i) For any $A \in \mathcal{O}$ and any $t \in \mathbb{R}, \pi_{X}\left(\tau_{\lambda}^{t}(A)\right)=\mathrm{e}^{\mathrm{i} t \mathcal{L}_{\lambda X}} \pi_{X}(A) \mathrm{e}^{-\mathrm{i} t \mathcal{L}_{\lambda X}}$.

(ii) $\mathcal{L}_{\lambda X}^{*} \Omega=0$.

Thus, for $A, B \in \mathcal{O}$ we have

$$
\omega_{X}^{(0)}\left(\tau_{\lambda}^{t}(A) B\right)=\left(\pi_{X}\left(A^{*}\right) \Omega, \mathrm{e}^{-\mathrm{i} t \mathcal{L}_{\lambda X}} \pi_{X}(B) \Omega\right)
$$

and hence the function

$$
z \mapsto \int_{0}^{\infty} \omega_{X}^{(0)}\left(\tau_{\lambda}^{t}(A) B\right) \mathrm{e}^{\mathrm{i} t z} \mathrm{~d} t=\mathrm{i}\left(\pi_{X}\left(A^{*}\right) \Omega,\left(z-\mathcal{L}_{\lambda X}\right)^{-1} \pi_{X}(B) \Omega\right),
$$

is analytic in the upper half-plane. The basic strategy of [JP2] is to show that for appropriate $A, B$ this function has a meromorphic continuation to a larger half-plane and that the behavior of $t \mapsto \omega_{X}^{(0)}\left(\tau_{\lambda}^{t}(A) B\right)$ as $t \rightarrow \infty$ is controlled by the poles of this continuation (the resonances) via the inverse Laplace transform.

Let $p_{j}=\mathrm{i} \partial_{s}$ be the generator of the group of translations on $\tilde{\mathfrak{h}}_{j}, P_{j}=\mathrm{d} \Gamma\left(p_{j}\right)$ its second quantization. Let $U_{j}(\theta)=\mathrm{e}^{-\mathrm{i} \theta P_{j}}=\Gamma\left(\mathrm{e}^{-\mathrm{i} \theta p_{j}}\right), \theta \in \mathbb{R}$, be the second quantization of this group and

$$
\begin{aligned}
V_{X}(\theta) & =\sum_{j} U_{j}(\theta) V_{j X} U_{j}(-\theta)=\sum_{j} \sigma_{x} \otimes I \otimes \tilde{\varphi}_{j}\left(\mathrm{e}^{-\mathrm{i} \theta p_{j}} \tilde{\alpha}_{j X}\right), \\
W_{X}(\theta) & =\sum_{j} U_{j}(\theta) W_{j X} U_{j}(-\theta)=\sum_{j} I \otimes \sigma_{x} \otimes \frac{1}{\sqrt{2}}(-I)^{N_{j}}\left(\tilde{a}_{j}^{*}\left(\mathrm{e}^{-\mathrm{i} \theta p_{j}} \tilde{\alpha}_{j X}\right)-\tilde{a}_{j}\left(\mathrm{e}^{-\mathrm{i} \theta p_{j}}\left(\mathrm{e}^{\left(X_{j}-\beta_{\mathrm{eq}}\right) s} \tilde{\alpha}_{j X}\right)\right)\right) .
\end{aligned}
$$


Lemma 3.1 There exist $\epsilon>0$ and $\delta^{\prime}>0$ such that the maps

$$
(X, \theta) \mapsto V_{X}(\theta), \quad(X, \theta) \mapsto W_{X}(\theta),
$$

extend to analytic operator-valued functions on $D_{\epsilon} \times I\left(\delta^{\prime}\right)$ satisfying

$$
\sup _{X \in D_{\epsilon}, \theta \in I\left(\delta^{\prime}\right)}\left(\left\|V_{X}(\theta)\right\|+\left\|W_{X}(\theta)\right\|\right)<\infty .
$$

In particular, one has

$$
\sup _{X \in D_{\epsilon},|t| \leq 1}\left\|\mathrm{e}^{\mathrm{i} t \mathcal{L}_{\lambda X}}\right\|<\infty
$$

Proof. The proof of the first part of this result is the same as the proof of Lemma 4.1 and Proposition 4.4 (iii) in [JP1]. The only additional fact needed is that for some $\epsilon>0$ and $\mu>0$ the function

$$
\mathbb{R} \times \mathbb{R} \ni(x, s) \mapsto w(x, s)=\left(\mathrm{e}^{-x s}+1\right)^{-1 / 2},
$$

has an analytic continuation to the region $O=\left\{z:\left|z-\beta_{\mathrm{eq}}\right|<\epsilon\right\} \times I(\mu)$ such that

$$
\sup _{(z, \theta) \in O}|w(z, \theta)|<\infty \text {. }
$$

Since $\mathcal{L}_{0}$ is self-adjoint, the bound (3.20) is a simple consequence of (3.19).

Let $N=\sum_{j} N_{j}$. For $X \in D_{\epsilon}$ and $\theta \in I\left(\delta^{\prime}\right)$ we set

$$
\begin{gathered}
\mathcal{L}_{0}(\theta)=\mathcal{L}_{0}+\theta N \\
\mathcal{L}_{\lambda X}(\theta)=\mathcal{L}_{0}(\theta)+\lambda\left(V_{X}(\theta)+W_{X}(\theta)\right) .
\end{gathered}
$$

The family of operators $\mathcal{L}_{\lambda X}(\theta), X \in D_{\epsilon}, \theta \in I\left(\delta^{\prime}\right)$, is a complex deformation of the family of operators $\mathcal{L}_{\lambda X}$, $X \in \mathbb{I}_{\epsilon}$. Note that $\mathcal{L}_{0 X}(\theta)=\mathcal{L}_{0}(\theta)$ is a normal operator which does not depend on $X$. The spectrum of $\mathcal{L}_{0}(\theta)$ consists of two simple eigenvalues \pm 2 , a doubly degenerate eigenvalue 0 and a sequence of $\operatorname{lines}\{x+\mathrm{i} n \operatorname{Im} \theta \mid x \in$ $\mathbb{R}, n \geq 1\}$. The next lemma is a consequence of Lemma 3.1 and regular perturbation theory and is deduced in the same way as the corresponding results in [JP1, JP2].

Proposition 3.2 There exist $\Lambda>0, \epsilon>0$ and $0<\mu<\delta^{\prime}$ such that for $|\lambda|<\Lambda,-\mu<\operatorname{Im} \theta<-3 \mu / 4$ and $X \in D_{\epsilon}$, the spectrum of $\mathcal{L}_{\lambda X}(\theta)$ is contained in the set

$$
\{z \mid \operatorname{Im} z>-\mu / 8\} \cup\{z \mid \operatorname{Im} z<-\mu / 2\} .
$$

The spectrum inside the half-plane $\{z \mid \operatorname{Im} z>-\mu / 8\}$ is discrete and, for $\lambda \neq 0$, consists of four simple eigenvalues $E_{j \lambda X}$ which do not depend on $\theta$ and are bounded analytic functions of $(\lambda, X) \in\{\lambda|| \lambda \mid<\Lambda\} \times D_{\epsilon}$. Moreover, $E_{0 \lambda X}=0$ and $\operatorname{Im} E_{j \lambda X}<0$ for $j=1,2,3, X \in D_{\epsilon}$, and $0<|\lambda|<\Lambda$. The corresponding eigenprojections $P_{j \lambda X}(\theta)$ are bounded analytic functions of the variables $(\lambda, X, \theta)$.

With regard to the results of [JP1, JP2], the only part of Proposition 3.2 that requires a comment are the relations $E_{0 \lambda X}=0$ and $\operatorname{Im} E_{j \lambda X}<0$ for $j=1,2,3$, which hold for $X \in D_{\epsilon}$ and $0<|\lambda|<\Lambda$. Regular perturbation theory and an explicit Fermi Golden Rule computation yield that the eigenvalues $E_{j \lambda X}, j=2,3$, which are respectively near \pm 2 , satisfy

$$
\begin{aligned}
& E_{2 \lambda X}=-2+\frac{\lambda^{2}}{2} \sum_{j}\left(-\mathrm{i} \pi\left\|\alpha_{j}(2)\right\|_{\mathfrak{H}_{j}}^{2}-\mathrm{PV} \int_{\mathbb{R}} \frac{\left\|\tilde{\alpha}_{j}(s)\right\|_{\mathfrak{H}_{j}}^{2}}{s-2} \mathrm{~d} s\right)+\lambda^{4} R_{2}(\lambda, X), \\
& E_{3 \lambda X}=2+\frac{\lambda^{2}}{2} \sum_{j}\left(-\mathrm{i} \pi\left\|\alpha_{j}(2)\right\|_{\mathfrak{H}_{j}}^{2}+\mathrm{PV} \int_{\mathbb{R}} \frac{\left\|\tilde{\alpha}_{j}(s)\right\|_{\mathfrak{H}_{j}}^{2}}{s-2} \mathrm{~d} s\right)+\lambda^{4} R_{3}(\lambda, X),
\end{aligned}
$$


where PV stands for Cauchy's principal value and the functions $R_{j}(\lambda, X), j=2,3$, are bounded and analytic for $X \in D_{\epsilon}$ and $|\lambda|<\Lambda$. Clearly, by choosing $\Lambda$ small enough, we have that $\operatorname{Im} E_{j \lambda X}<0$ for $j=2,3, X \in D_{\epsilon}$, and $0<|\lambda|<\Lambda$. The eigenvalues $E_{j \lambda X}, j=0,1$, which are near 0 , are the eigenvalues of a $2 \times 2$-matrix $\Sigma_{\lambda X}$ which has the form

$$
\Sigma_{\lambda X}=\lambda^{2} \Sigma_{2}(X)+\lambda^{4} R(\lambda, X)
$$

where the matrix-valued function $R(\lambda, X)$ is analytic and bounded for $X \in D_{\epsilon}$ and $|\lambda|<\Lambda$ and

$$
\Sigma_{2}(X)=-\mathrm{i} \pi \sum_{j}\left\|\alpha_{j}(2)\right\|_{\mathfrak{H}_{j}}^{2} \frac{1}{2 \cosh \beta_{j}}\left[\begin{array}{cc}
\mathrm{e}^{\beta_{j}} & -\mathrm{e}^{-\beta_{j}} \\
-\mathrm{e}^{\beta_{j}} & \mathrm{e}^{-\beta_{j}}
\end{array}\right], \quad \beta_{j}=\beta_{\mathrm{eq}}-X_{j}
$$

The eigenvalues of $\Sigma_{2}(X)$ are 0 and $-\mathrm{i} \pi \sum_{j}\left\|\alpha_{j}(2)\right\|_{\mathfrak{H}_{j}}^{2}$, and we conclude that for $\Lambda$ small enough the eigenvalues $E_{0 \lambda X}$ and $E_{1 \lambda X}$ are analytic functions, that $E_{0 \lambda X} \neq E_{1 \lambda X}$ for $\lambda \neq 0$, and that $\operatorname{Im} E_{1 \lambda X}<0$ for $X \in D_{\epsilon}$, $0<|\lambda|<\Lambda$. By construction of the $C$-Liouvillean, $E_{0 \lambda X}=0$ for $X$ real. Hence, by analyticity, $E_{0 \lambda X}=0$ for $X \in D_{\epsilon}$ and $|\lambda|<\Lambda$.

The next technical result we need is:

Proposition 3.3 There exist $\Lambda>0, \epsilon>0$, and $\mu>0$ such that for all $|\lambda|<\Lambda$, all $\theta$ in the strip $-\mu<\operatorname{Im} \theta<$ $-3 \mu / 4$ and all $\Psi \in \mathcal{H}$, the functions defined by

$$
F_{+}(z)=\sup _{X \in D_{\epsilon}}\left\|\left(z-\mathcal{L}_{\lambda X}(\theta)\right)^{-1} \Psi\right\|, \quad F_{-}(z)=\sup _{X \in D_{\epsilon}}\left\|\left(\bar{z}-\mathcal{L}_{\lambda X}(\theta)^{*}\right)^{-1} \Psi\right\|
$$

satisfy

$$
\int_{\mathbb{R}}\left|F_{ \pm}(x \pm \mathrm{i} \mu)\right|^{2} \mathrm{~d} x \leq \frac{16 \pi}{\mu}\|\Psi\|^{2}
$$

and

$$
\lim _{|x| \rightarrow \infty} F_{ \pm}(x+\mathrm{i} \eta)=0
$$

for all $|\eta| \leq \mu / 4$.

Proof. We only deal with $F_{+}(z)$, the other case is similar. We start with $\Lambda, \epsilon$, and $\mu$ as in Proposition 3.2 and set

$$
Q_{\mu}=(\mathbb{R}+\mathrm{i} \mu / 4) \cup(\mathbb{R}-\mathrm{i} \mu / 4) \cup\{z \in \mathbb{C}|| \operatorname{Re} z|\geq 2+\mu / 4,| \operatorname{Im} z \mid \leq \mu / 4\}
$$

Since $\mathcal{L}_{0}(\theta)$ is normal and $\operatorname{dist}\left(Q_{\mu}, \sigma\left(\mathcal{L}_{0}(\theta)\right)\right) \geq \mu / 4$ for $\operatorname{Im} \theta \leq-3 \mu / 4$, the spectral theorem yields that

$$
\sup _{z \in Q_{\mu}, \operatorname{Im} \theta \leq-3 \mu / 4}\left\|\left(z-\mathcal{L}_{0}(\theta)\right)^{-1}\right\| \leq \frac{4}{\mu} .
$$

The estimate

$$
\int_{\mathbb{R}}\left\|\left(x \pm \mathrm{i} \mu / 4-\mathcal{L}_{0}(\theta)\right)^{-1} \Psi\right\|^{2} \mathrm{~d} x \leq \frac{4 \pi\|\Psi\|^{2}}{\mu},
$$

holds for all $\Psi \in \mathcal{H}$, and the dominated convergence theorem yields

$$
\lim _{|z| \rightarrow \infty, z \in Q_{\mu}}\left\|\left(z-\mathcal{L}_{0}(\theta)\right)^{-1} \Psi\right\|=0 .
$$

We further impose that $\Lambda$ and $\mu$ satisfy

$$
\sup _{X \in D_{\epsilon},-\mu<\operatorname{Im} \theta<0}\left\|V_{X}(\theta)+W_{X}(\theta)\right\| \leq \frac{\mu}{8 \Lambda} .
$$


The resolvent identity yields

$$
\left(z-\mathcal{L}_{\lambda X}(\theta)\right)^{-1}=G(z, \lambda, X, \theta)\left(z-\mathcal{L}_{0}(\theta)\right)^{-1}
$$

where

$$
G=G(z, \lambda, X, \theta)=\left(I-\lambda\left(z-\mathcal{L}_{0}(\theta)\right)^{-1}\left(V_{X}(\theta)+W_{X}(\theta)\right)\right)^{-1} .
$$

The estimate (3.24) yields

$$
\sup \|G\| \leq 2,
$$

where the supremum is taken over $z \in Q_{\mu},|\lambda|<\Lambda, X \in D_{\epsilon}$, and $\theta$ in the strip $-\mu<\operatorname{Im} \theta<-3 \mu / 4$. Hence, for $z \in Q_{\mu}$,

$$
\sup _{X \in D_{\epsilon}}\left\|\left(z-\mathcal{L}_{\lambda X}(\theta)\right)^{-1} \Psi\right\| \leq 2\left\|\left(z-\mathcal{L}_{0}(\theta)\right)^{-1} \Psi\right\|
$$

and (3.25), (3.26) yield (3.22), (3.23).

Assumption (A2) ensures that there is $\epsilon>0$ such that the operators

$$
V(X, u)=\sum_{j=1}^{M} \sigma_{x} \otimes I \otimes \frac{1}{\sqrt{2}}\left(\tilde{a}_{j}^{*}\left(\mathrm{e}^{-u\left(1-X_{j} / \beta_{\mathrm{eq}}\right) s} \tilde{\alpha}_{j X}\right)+\tilde{a}_{j}\left(\mathrm{e}^{u\left(1-X_{j} / \beta_{\mathrm{eq}}\right) s} \tilde{\alpha}_{j X}\right)\right),
$$

acting on $\mathcal{H}$ are well-defined continuous functions of $(X, u) \in \mathbb{I}_{\epsilon} \times\left[0, \beta_{\text {eq }}\right]$ satisfying

$$
\sup _{(X, u) \in \mathbb{I}_{\epsilon} \times\left[0, \beta_{\mathrm{eq}}\right]}\|V(x, u)\|<\infty .
$$

If we set

$$
\mathcal{G}_{\lambda X}=\mathbb{1}+\sum_{n \geq 1}\left(-\beta_{\mathrm{eq}}\right)^{n} \int_{0 \leq t_{n} \leq \cdots \leq t_{1} \leq 1}\left(\lambda V\left(X, \beta_{\mathrm{eq}} t_{n}\right)+\pi_{X}\left(H_{\mathcal{S}}\right)\right) \cdots\left(\lambda V\left(X, \beta_{\mathrm{eq}} t_{1}\right)+\pi_{X}\left(H_{\mathcal{S}}\right)\right) \mathrm{d} t_{1} \cdots \mathrm{d} t_{n},
$$

then the Araki perturbation theory [Ar, BR2, DJP] yields that the reference state $\omega_{\lambda X}$ can be written as

$$
\omega_{\lambda X}(A)=\frac{\left(\Omega, \pi_{X}(A) \mathcal{G}_{\lambda X} \Omega\right)}{\left(\Omega, \mathcal{G}_{\lambda X} \Omega\right)} .
$$

Consider the unitary group

$$
U(\theta)=\mathrm{e}^{-\mathrm{i} \theta \sum_{j} P_{j}},
$$

on $\mathcal{H}$.

Proposition 3.4 There exist $\epsilon>0$ and $\mu>0$ such that:

(1) The function

$$
\mathbb{I}_{\epsilon} \times \mathbb{R} \ni(X, \theta) \mapsto U(\theta) \mathcal{G}_{\lambda X} \Omega \in \mathcal{H},
$$

extends to a bounded analytic $\mathcal{H}$-valued function in the region $D_{\epsilon} \times I(\mu)$ for all $\lambda \in \mathbb{R}$. We denote this analytic extension by $\Omega_{\lambda X \theta}$.

(2) The functions

$$
\mathbb{I}_{\epsilon} \times \mathbb{R} \ni(X, \theta) \mapsto U(\theta) \pi_{X}\left(\Phi_{j}\right) \Omega \in \mathcal{H},
$$

extend to bounded analytic $\mathcal{H}$-valued functions in the region $D_{\epsilon} \times I(\mu)$. We denote this analytic extensions by $\Psi_{j X \theta}$. 
Proof. We sketch the proof of (1). The proof of (2) is similar and simpler.

For $(X, u, \theta) \in \mathbb{I}_{\epsilon} \times\left[0, \beta_{\text {eq }}\right] \times \mathbb{R}$ we set

$$
\begin{aligned}
V_{\theta}(X, u) & =U(\theta) V(X, u) U(\theta)^{*} \\
& =\sum_{j=1}^{M} \sigma_{x} \otimes I \otimes \frac{1}{\sqrt{2}}\left(\tilde{a}_{j}^{*}\left(\mathrm{e}^{-\mathrm{i} \theta p_{j}} \mathrm{e}^{-u\left(1-X_{j} / \beta_{\mathrm{eq}}\right) s} \tilde{\alpha}_{j X}\right)+\tilde{a}_{j}\left(\mathrm{e}^{-\mathrm{i} \theta p_{j}} \mathrm{e}^{u\left(1-X_{j} / \beta_{\mathrm{eq}}\right) s} \tilde{\alpha}_{j X}\right)\right) .
\end{aligned}
$$

Since $U(\theta) \Omega=\Omega$, we can write $U(\theta) \mathcal{G}_{\lambda X} \Omega=\mathcal{G}_{\lambda X \theta} \Omega$ where $\mathcal{G}_{\lambda X \theta}$ is obtained by replacing $V(X, u)$ by $V_{\theta}(X, u)$ in the definition of $\mathcal{G}_{\lambda X}$. It is easy to see for any $\epsilon>0, \mu>0$ and $\rho>0$ the entire analytic function $g(u, z, s)=$ $\mathrm{e}^{u\left(1-z / \beta_{\text {eq }}\right) s}$ satisfies

$$
\sup _{|u|<(1+\rho) \beta_{\mathrm{eq}},|z|<\epsilon,|\operatorname{Im} s|<\mu}\left|\frac{g(u, z, s)}{\cosh (l s)}\right|<\infty,
$$

where $l=(1+\rho)\left(\epsilon+\beta_{\mathrm{eq}}\right)$. Let $a>\beta_{\mathrm{eq}}$ be as in Assumption (A2). Choose $\rho$ and $\epsilon$ such that $l<a$. Since by (A2) one has $\cosh (a s) \tilde{\alpha}_{j X} \in H_{j}^{2}(\delta)$, it follows that $V_{\theta}(X, u)$ has a bounded analytic extension to the set

$$
\left\{(X, u, \theta)\left|X \in D_{\epsilon}, u \in \mathbb{C},\right| u\left|<(1+\rho) \beta_{\text {eq }},\right| \operatorname{Im} \theta \mid<\mu\right\} .
$$

This yields the statement.

Proof of Theorem 2.2. We choose $\Lambda>0, \epsilon>0$, and $\mu>0$ sufficiently small so that the statements in Propositions 3.2, 3.3 and 3.4 hold. Combining (3.18) and (3.27) we can write

$$
\omega_{\lambda X}\left(\tau_{\lambda}^{t}\left(\Phi_{j}\right)\right)=\frac{\left(\pi_{X}\left(\Phi_{j}\right) \Omega, \mathrm{e}^{-\mathrm{i} t \mathcal{L}_{\lambda X}} \mathcal{G}_{\lambda X} \Omega\right)}{\left(\Omega, \mathcal{G}_{\lambda X} \Omega\right)} .
$$

Since for $X \in I_{\epsilon}$

$$
\left(\Omega, \mathcal{G}_{\lambda X} \Omega\right)=\left\|\mathrm{e}^{-\beta_{\mathrm{eq}}\left(\sum_{j}\left(1-X_{j} / \beta_{\mathrm{eq}}\right) \mathcal{L}_{j}+\pi_{X}\left(\lambda V+H_{\mathcal{S}}\right)\right) / 2} \Omega\right\|^{2}>0,
$$

by Proposition 3.4 (and by possibly taking $\epsilon$ smaller), the function $X \mapsto\left(\Omega, \mathcal{G}_{\lambda X} \Omega\right)$ extends to an analytic function in the region $D_{\epsilon}$ such that

$$
\inf _{X \in D_{\epsilon}}\left|\left(\Omega, \mathcal{G}_{\lambda X} \Omega\right)\right|>0
$$

Thus, it suffices to consider the numerator in (3.28). For $\operatorname{Im} z>0$ we set

$$
D_{X}(z)=\mathrm{i}\left(\pi_{X}\left(\Phi_{j}\right) \Omega,\left(z-\mathcal{L}_{\lambda X}\right)^{-1} \mathcal{G}_{\lambda X} \Omega\right) .
$$

For $|\lambda|<\Lambda, X \in \mathbb{I}_{\epsilon}$ and $-\mu<\operatorname{Im} \theta<-3 \mu / 4$ one has

$$
D_{X}(z)=\mathrm{i}\left(\Psi_{j X \bar{\theta}},\left(z-\mathcal{L}_{\lambda X}(\theta)\right)^{-1} \Omega_{\lambda X \theta}\right),
$$

which, by Proposition 3.2, has a meromorphic extension to the half-plane $\{\operatorname{Im} z>-\mu / 2\}$. For $\alpha>0$ denote by $\Gamma_{\alpha}$ the boundary of the rectangle with vertices $\pm \alpha \pm \mathrm{i} \mu / 4$. For large enough $\alpha$ one has

$$
I_{X}(t)=\oint_{\Gamma_{\alpha}} \mathrm{e}^{-\mathrm{i} t z} D_{X}(z) \frac{\mathrm{d} z}{2 \pi \mathrm{i}}=\mathrm{i} \sum_{j=0}^{3}\left(\Psi_{j X \bar{\theta}}, P_{j \lambda X}(\theta) \Omega_{\lambda X \theta}\right) \mathrm{e}^{-\mathrm{i} t E_{j \lambda X}} .
$$

Denote by $S_{\alpha}$ the part of the above contour integral corresponding to the two vertical sides of $\Gamma_{\alpha}$. It follows from the dominated convergence theorem and Proposition 3.3 that $\lim _{\alpha \rightarrow \infty} S_{\alpha}=0$. Since by Proposition 3.3 the function $x \mapsto D_{X}(x+\mathrm{i} \mu / 4)$ is in $L^{2}(\mathbb{R}, \mathrm{d} x)$ it follows from the Plancherel theorem that there exists a sequence $\alpha_{n}$ such that

$$
\lim _{n} \int_{-\alpha_{n}}^{\alpha_{n}} \mathrm{e}^{-\mathrm{i} t(x+\mathrm{i} \mu / 4)} D_{X}(x+\mathrm{i} \mu / 4) \frac{\mathrm{d} x}{2 \pi}=\left(\pi_{X}\left(\Phi_{j}\right) \Omega, \mathrm{e}^{-\mathrm{i} t \mathcal{L}_{\lambda X}} \mathcal{G}_{\lambda X} \Omega\right),
$$


for Lebesgue almost all $t>0$. Integration by parts and (3.23) yield that for $t>0$

$$
\lim _{n} \int_{-\alpha_{n}}^{\alpha_{n}} \mathrm{e}^{-\mathrm{i} t(x-\mathrm{i} \mu / 4)} D_{X}(x-\mathrm{i} \mu / 4) \frac{\mathrm{d} x}{2 \pi}=\int_{-\infty}^{\infty} \mathrm{e}^{-\mathrm{i} t(x-\mathrm{i} \mu / 4)} D_{X}^{\prime}(x-\mathrm{i} \mu / 4) \frac{\mathrm{d} x}{2 \pi \mathrm{i} t},
$$

where $D_{X}^{\prime}(z)$ denotes the derivative of $D_{X}(z)$ with respect to $z$. Combining these facts we obtain the identity

$$
\begin{aligned}
& \left(\pi_{X}\left(\Phi_{j}\right) \Omega, \mathrm{e}^{-\mathrm{i} t \mathcal{L}_{\lambda X}} \mathcal{G}_{\lambda X} \Omega\right)=\sum_{j=0}^{3}\left(\Psi_{j X \bar{\theta}}, P_{j \lambda X}(\theta) \Omega_{\lambda X \theta}\right) \mathrm{e}^{-\mathrm{i} t E_{j \lambda X}} \\
& -\frac{\mathrm{e}^{-\mu t / 4}}{2 \pi t} \int_{-\infty}^{\infty} \mathrm{e}^{-\mathrm{i} t x}\left(\Psi_{j X \bar{\theta}},\left(x-\mathrm{i} \mu / 4-\mathcal{L}_{\lambda X}(\theta)\right)^{-2} \Omega_{\lambda X \theta}\right) \mathrm{d} x,
\end{aligned}
$$

which holds for Lebesgue for almost all $t>0$. By Proposition 3.3 the integrand on the right hand side of (3.29) is in $L^{1}(\mathbb{R}, \mathrm{d} x)$. Hence, both side of this identity are continuous functions of $t$ and (3.29) holds for all $t>0$. By Propositions 3.2 and 3.4 both terms on the right hand side of (3.29) have analytic extensions to $X \in D_{\epsilon}$ which are bounded uniformly in $X$ and $t \geq 1$. The bound (3.20) and Proposition 3.4 yield that

$$
\sup _{X \in D_{\epsilon}, t \in[0,1]}\left|\left(\Psi_{j X 0}, \mathrm{e}^{-i t \mathcal{L}_{\lambda X}} \Omega_{\lambda X 0}\right)\right|<\infty,
$$

and the result follows.

\section{Proof of Theorem 1.4}

In Theorem 1.2 we have established that for given $\lambda, X \mapsto \omega_{\lambda X+}\left(\Phi_{j}\right)$ is analytic near zero. In fact, a stronger result holds.

Theorem 4.1 Assume that (A1)-(A3) hold. Then there is $\Lambda>0$ and $\epsilon>0$ such that the maps

$$
(\lambda, X) \mapsto \omega_{\lambda X+}\left(\Phi_{j}\right),
$$

extend to analytic functions on $\{\lambda|| \lambda \mid<\Lambda\} \times D_{\epsilon}$.

Proof. By the construction of the NESS $\omega_{\lambda X+}$,

$$
\omega_{\lambda X+}\left(\Phi_{j}\right)=\left(\Omega, P_{0 \lambda X}(\theta) U(\theta) \pi_{X}\left(\Phi_{j}\right) \Omega\right),
$$

where $-\mu<\operatorname{Im} \theta<-3 \mu / 4$ and $P_{0 \lambda X}(\theta)$ and $\mu$ are as in Proposition 3.2. The analyticity of $P_{0 \lambda X}(\theta)$ and Part (2) of Proposition 3.4 yield the statement.

Theorem 4.1 yields that the function $\lambda \mapsto L_{\lambda j i}$ is analytic near zero. To compute the leading term in its power expansion we argue as follows.

By the relation (1.15) established in [JP2, JP3],

$$
\omega_{\lambda X+}\left(\Phi_{j}\right)=\lambda^{2} \omega_{\mathcal{S} X+}\left(\bar{\Phi}_{j X}\right)+O\left(\lambda^{3}\right),
$$

where the remainder is uniform in $X$. Hence, (1.6) holds and

$$
L_{j i}^{(2)}=\left.\partial_{X_{i}} \omega_{\mathcal{S} X+}\left(\bar{\Phi}_{j X}\right)\right|_{X=0} .
$$


Let $\mathcal{D} \subset \mathcal{O}_{\mathcal{S}}$ be the set of observables which are diagonal in the eigenbasis $\left\{e_{+}, e_{-}\right\}$of $H_{\mathcal{S}}$. The generators $K_{X}$ and $K_{X_{j}}$ preserve $\mathcal{D}$. The vector space $\mathcal{D}$ is naturally identified with $\mathbb{C}^{2}$. After this identification, $K_{X}=\mathrm{i} \Sigma_{2}(X)^{*}$, where $\Sigma_{2}(X)$ is given by (3.21), and

$$
K_{X_{j}}=-\frac{\pi\left\|\alpha_{j}(2)\right\|_{\mathfrak{H}_{j}}^{2}}{2 \cosh \beta_{j}}\left[\begin{array}{cc}
\mathrm{e}^{\beta_{j}} & -\mathrm{e}^{\beta_{j}} \\
-\mathrm{e}^{-\beta_{j}} & \mathrm{e}^{-\beta_{j}}
\end{array}\right], \quad \beta_{j}=\beta_{\mathrm{eq}}-X_{j} .
$$

These relations between the generators $K_{X}, K_{X_{j}}$ and the Fermi Golden Rule for the resonances of the $C$ Liouvillean are quite general—for the proofs and additional information we refer the reader to [DJ]. Hence,

$$
\bar{\Phi}_{j X}=K_{X_{j}}\left[\begin{array}{c}
1 \\
-1
\end{array}\right]=-\frac{\pi\left\|\alpha_{j}(2)\right\|_{\mathfrak{H}_{j}}^{2}}{\cosh \beta_{j}}\left[\begin{array}{c}
\mathrm{e}^{\beta_{j}} \\
-\mathrm{e}^{-\beta_{j}}
\end{array}\right] .
$$

The density matrix describing $\omega_{\mathcal{S} X+}$ (which we denote by the same letter) is also diagonal in the basis $\left\{e_{+}, e_{-}\right\}$and the vector in $\mathbb{C}^{2}$ associated to its diagonal elements is the eigenvector of $\Sigma_{2}(X)$ corresponding to the eigenvalue 0. Hence,

$$
\omega_{\mathcal{S} X+}=\left(\sum_{j}\left\|\alpha_{j}(2)\right\|_{\mathfrak{H}_{j}}^{2}\right)^{-1}\left[\begin{array}{c}
\sum_{j} \frac{\left\|\alpha_{j}(2)\right\|_{\mathfrak{H}_{j}}^{2} \mathrm{e}^{-\beta_{j}}}{2 \cosh \beta_{j}} \\
\sum_{j} \frac{\left\|\alpha_{j}(2)\right\|_{\mathfrak{H}_{j}}^{2} \mathrm{e}^{\beta_{j}}}{2 \cosh \beta_{j}}
\end{array}\right],
$$

and we get

$$
\omega_{\mathcal{S} X+}\left(\bar{\Phi}_{j X}\right)=\pi\left(\sum_{k}\left\|\alpha_{k}(2)\right\|_{\mathfrak{H}_{k}}^{2}\right)^{-1} \frac{\left\|\alpha_{j}(2)\right\|_{\mathfrak{H}_{j}}^{2}}{\cosh \beta_{j}} \sum_{k}\left\|\alpha_{k}(2)\right\|_{\mathfrak{H}_{k}}^{2} \frac{\sinh \left(\beta_{k}-\beta_{j}\right)}{\cosh \beta_{k}}
$$

It follows that for $i \neq j$,

$$
L_{j i}^{(2)}=\left.\partial_{X_{i}} \omega_{\mathcal{S} X+}\left(\bar{\Phi}_{j X}\right)\right|_{X=0}=-\frac{\pi}{\left(\cosh \beta_{\mathrm{eq}}\right)^{2}} \frac{\left\|\alpha_{i}(2)\right\|_{\mathfrak{H}_{i}}^{2}\left\|\alpha_{j}(2)\right\|_{\mathfrak{H}_{j}}^{2}}{\sum_{k}\left\|\alpha_{k}(2)\right\|_{\mathfrak{H}_{k}}^{2}} .
$$

Since $\sum_{j} \omega_{\mathcal{S} X+}\left(\bar{\Phi}_{j X}\right)=0$ we can conclude that $L_{i i}^{(2)}=-\sum_{j \neq i} L_{j i}^{(2)}$.

Finally, we remark that the formula (4.30) yields that

$$
\overline{\mathrm{Ep}}=\frac{\pi}{2}\left(\sum_{k}\left\|\alpha_{k}(2)\right\|_{\mathfrak{H}_{k}}^{2}\right)^{-1} \sum_{i, j} \frac{\left\|\alpha_{i}(2)\right\|_{\mathfrak{H}_{i}}^{2}\left\|\alpha_{j}(2)\right\|_{\mathfrak{H}_{j}}^{2}}{\cosh \beta_{i} \cosh \beta_{j}}\left(\beta_{i}-\beta_{j}\right) \sinh \left(\beta_{i}-\beta_{j}\right) .
$$

Clearly, $\overline{\mathrm{Ep}}>0$ whenever $\beta_{j}$ 's are not all equal.

\section{Proofs of Theorems 1.5 and 1.6.}

In this section we use the notational conventions of Subsection 1.2.

Proof of Theorem 1.5. The only part that requires a proof is (3). We only sketch the argument. Let $\vec{\beta}_{0}=$ $\left(\beta_{10}, \cdots, \beta_{M 0}\right)$ be a given point and $O_{\epsilon}=\left\{\vec{\beta} \in \mathbb{C}^{M}|| \vec{\beta}-\vec{\beta}_{0} \mid<\epsilon\right\}$. Arguing as in the proof of Lemma 3.1 one shows that there exists $\epsilon>0$ and $\delta^{\prime}>0$ such that such that the maps

$$
(\vec{\beta}, \theta) \mapsto V_{\vec{\beta}}(\theta), \quad(\vec{\beta}, \theta) \mapsto W_{\vec{\beta}}(\theta),
$$


extend to analytic operator-valued functions on $O_{\epsilon} \times I\left(\delta^{\prime}\right)$ satisfying

$$
\sup _{\vec{\beta} \in O_{\epsilon}, \theta \in I\left(\delta^{\prime}\right)}\left(\left\|V_{\vec{\beta}}(\theta)\right\|+\left\|W_{\vec{\beta}}(\theta)\right\|\right)<\infty .
$$

This implies that Proposition 3.2 holds with $D_{\epsilon}$ replaced with $O_{\epsilon}$ (of course, the index $X$ is also replaced by $\vec{\beta})$. Note that $\Lambda$ depends on the $\epsilon$. Complementing the construction in [JP2] with arguments used in the proof of Proposition 3.4 one easily shows that there exists a norm-dense $*$-algebra $\mathcal{O}_{0}$ of $\mathcal{O}$ such that:

(a) $\mathcal{O}_{0}$ does not depend on the choice of $\vec{\beta}_{0}$;

(b) $\Phi_{j} \in \mathcal{O}_{0}$;

(c) for all $A \in \mathcal{O}_{0}$ the functions

$$
(\vec{\beta}, \theta) \mapsto U(\theta) \pi_{\vec{\beta}}(A) \Omega \in \mathcal{H},
$$

extend to bounded analytic $\mathcal{H}$-valued functions in the region $O_{\epsilon} \times I(\mu)$. The representation

$$
\omega_{\lambda \vec{\beta}+}(A)=\left(\Omega, P_{0 \lambda \vec{\beta}}(\theta) U(\theta) \pi_{\vec{\beta}}(A) \Omega\right),
$$

where $-\mu<\operatorname{Im} \theta<-3 \mu / 4$ and $P_{0 \lambda \vec{\beta}}(\theta)$ and $\mu$ are as in the analog of Proposition 3.2, yields the following statement: For any given $\vec{\beta}_{0} \in \mathbb{I}_{\gamma_{1} \gamma_{2}}$ there exists $\Lambda$ and $\epsilon$ such that the function

$$
(\lambda, \vec{\beta}) \mapsto \omega_{\lambda \vec{\beta}+}(A),
$$

extends to an analytic functions on $\{\lambda|| \lambda \mid<\Lambda\} \times O_{\epsilon}$ for all $A \in \mathcal{O}_{0}$. This fact and the compactness of $I_{\gamma_{1} \gamma_{2}}$ yield the statement.

Proof of Theorem 1.6. By Remark 1 after Theorem 1.6, it suffices to establish Part (1). By Remark 2, it suffices to show that there exists $\delta>0$ and $\Lambda>0$ such that for $0<|\lambda|<\Lambda$

$$
\operatorname{Ep}\left(\omega_{\lambda \vec{\beta}+}\right)>0
$$

for $\vec{\beta} \in \mathbb{I}_{\gamma_{1} \gamma_{2}}$ satisfying $0<\sum_{i, j}\left|\beta_{i}-\beta_{j}\right|<\delta$.

Let $\vec{\beta}_{0}=\left(\beta_{0}, \cdots, \beta_{0}\right)$ be a given point on the diagonal of $\mathbb{I}_{\gamma_{1} \gamma_{2}}$. We set

$$
O_{\delta}=\left\{\vec{\beta} \in \mathbb{C}^{M}\left|\sum_{j}\right| \beta_{j}-\beta_{0} \mid<\delta\right\},
$$

and $\mathbb{I}_{\delta}=O_{\delta} \cap \mathbb{R}^{M}$. One can choose $\Lambda$ and $\delta$ such that $(\lambda, \vec{\beta}) \mapsto \operatorname{Ep}\left(\omega_{\lambda \vec{\beta}+}\right)$ is an analytic function on $\{|\lambda|<$ $\Lambda\} \times O_{\delta}$. We set

$$
Y_{\vec{\beta}}=\left(\beta_{2}-\beta_{1}, \cdots, \beta_{M}-\beta_{1}\right) .
$$

Setting $\beta_{1}=\beta_{\text {eq }}$ one deduces from the formula (4.31) and the Taylor series for $\operatorname{Ep}\left(\omega_{\lambda \vec{\beta}+}\right)$ (use that $\operatorname{Ep}\left(\omega_{\lambda \vec{\beta}+}\right)$ and $\partial_{\beta_{i}} \operatorname{Ep}\left(\omega_{\lambda \vec{\beta}+}\right)$ vanish when all $\beta_{j}$ are equal) that there exists $(M-1) \times(M-1)$-matrix valued functions $A(\vec{\beta})$ and $B(\lambda, \vec{\beta})$ such that:

(a) $A(\vec{\beta})$ is analytic for $\vec{\beta} \in O_{\delta}$ and strictly positive for $\vec{\beta}$ real;

(b) $B(\lambda, \vec{\beta})$ is analytic and bounded on $\{|\lambda|<\Lambda\} \times O_{\epsilon}$;

(c)

$$
\operatorname{Ep}\left(\omega_{\lambda \vec{\beta}+}\right)=\lambda^{2}\left(Y_{\vec{\beta}}, A(\vec{\beta}) Y_{\vec{\beta}}\right)+\lambda^{3}\left(Y_{\vec{\beta}}, B(\lambda, \vec{\beta}) Y_{\vec{\beta}}\right)
$$

By choosing $\Lambda$ small enough we can ensure that for all $\vec{\beta} \in \mathbb{I}_{\delta}$ and $|\lambda|<\Lambda$,

$$
\left.\left(Y_{\vec{\beta}}, A(\vec{\beta}) Y_{\vec{\beta}}\right)>\mid \lambda\left(Y_{\vec{\beta}}, B(\lambda, \vec{\beta})\right) Y_{\vec{\beta}}\right) \mid \text {. }
$$

This yields that $\operatorname{Ep}\left(\omega_{\lambda \vec{\beta}+}\right)>0$ for $0<|\lambda|<\Lambda$ and $\vec{\beta} \in \mathbb{I}_{\delta}$ satisfying $Y_{\vec{\beta}} \neq 0$. This local result combined with an obvious compactness argument yields the statement. 


\section{References}

[Ar] Araki, H.: Relative Hamiltonians for faithful normal states of a von Neumann algebra. Publ. R.I.M.S., Kyoto Univ. 9, 165 (1973).

[AH] Araki, H., Ho, T.G: Asymptotic time evolution of a partitioned infinite two-sided isotropic $X Y$-chain. Tr. Mat. Inst. Steklova, 228 Probl. Sovrem. Mat. Fiz., 203, (2000); translation in Proc. Steklov Inst. Math. 228, 191, (2000).

[AP] Aschbacher, W., Pillet, C-A.: Non-equilibrium steady states of the $X Y$ chain. J. Stat. Phys. 12, 1153 (2003).

[AJPP1] Aschbacher, W., Jakšić, V., Pautrat, Y., Pillet, C.-A.: Topics in non-equilibrium quantum statistical mechanics. In Open Quantum Systems III. Lecture Notes of the Summer School on Open Quantum Systems held in Grenoble, June 16-July 4, 2003. To be published in Lecture Notes in Mathematics, Springer, New York.

[AJPP2] Aschbacher, W., Jakšić, V., Pautrat, Y., Pillet, C.-A.: Transport properties of ideal Fermi gases (in preparation).

[AW] Araki, H., Wyss, W.: Representations of canonical anti-commutation relations. Helv. Phys. Acta 37, 136 (1964).

[BGKS] Bouclet, J.M., Germinet, F., Klein, A., and Schenker, J.H.: Linear response theory for magnetic Schrödinger operators in disordered media. J. Funct. Anal. (in press).

[BR1] Bratteli, O., Robinson, D. W.: Operator Algebras and Quantum Statistical Mechanics 1. Springer-Verlag, Berlin (1987).

[BR2] Bratteli, O., Robinson, D. W.: Operator Algebras and Quantum Statistical Mechanics 2. Second edition, SpringerVerlag, Berlin (1996).

[Da] Davies, E.B.: Markovian master equations. Commun. Math. Phys. 39, 91 (1974).

[De] Dereziński, J.: Fermi Golden Rule and open quantum systems. In Open Quantum Systems III. Lecture Notes of the Summer School on Open Quantum Systems held in Grenoble, June 16-July 4, 2003. To be published in Lecture Notes in Mathematics, Springer, New York.

[DJ] Dereziński, J., Jakšić, V.: On the nature of Fermi Golden Rule for open quantum systems. J. Stat. Phys. 116, 411 (2004).

[DJP] Dereziński, J., Jakšić, V., Pillet, C.-A.: Perturbation theory of $W^{*}$-dynamics, KMS-states and Liouvilleans. Rev. Math. Phys. 15, 447 (2003).

[FMU] Fröhlich, J., Merkli, M., Ueltschi, D.: Dissipative transport: thermal contacts and tunneling junctions. Ann. Henri Poincaré 4, 897 (2004).

[GVV] Goderis, D., Verbeure, A., Vets, P.: About the exactness of the linear response theory. Commun. Math. Phys. 136, 265 (1991).

[JOP1] Jakšić, V., Ogata, Y., Pillet, C.-A.: The Green-Kubo formula and the Onsager reciprocity relations in quantum statistical mechanics. Submitted.

[JOP2] Jakšić, V., Ogata, Y., Pillet, C.-A.: Linear response theory in quantum statistical mechanics. Preprint.

[JOPP] Jakšić, V., Ogata, Y., Pautrat, Y., Pillet, C.-A.: In preparation.

[JP1] Jakšić, V., Pillet, C-A.: On a model for quantum friction II. Fermi's golden rule and dynamics at positive temperature. Commun. Math. Phys. 176, 619 (1996).

[JP2] Jakšić, V., Pillet, C.-A.: Non-equilibrium steady states for finite quantum systems coupled to thermal reservoirs. Commun. Math. Phys. 226, 131 (2002).

[JP3] Jakšić, V., Pillet, C.-A.: Mathematical theory of non-equilibrium quantum statistical mechanics. J. Stat. Phys. 108, 787 (2002).

[JP4] Jakšić, V., Pillet, C.-A.: In preparation.

[JP5] Jakšić, V., Pillet, C.-A.: A note on the entropy production formula. Contemp. Math. 327, 175 (2003).

[KTH] Kubo, R., Toda, M., Hashitsune, N.: Statistical Physics II. Second edition, Springer-Verlag, Berlin (1991).

[LeSp] Lebowitz, J., Spohn, H.: Irreversible thermodynamics for quantum systems weakly coupled to thermal reservoirs. Adv. Chem. Phys. 39, 109 (1978). 
[MMS] Merkli, M., Mueck, M., Sigal, I.M.: Instability of equilibrium states for coupled heat reservoirs at different temperatures. Preprint.

[NVW] Naudts, J., Verbeure, A., Weder, R.: Linear response theory and the KMS condition. Commun. Math. Phys. 44, 87 (1975).

[RBT] Rey-Bellet, L., Thomas, L.E.: Fluctuations of the entropy production in anharmonic chains. Ann. Henri Poinc. 3, 483 (2002).

[Si] Simon, B.: Fifteen problems in mathematical physics. Perspectives in mathematics, Birkhäuser, Basel, 423 (1984).

[Sp] Spohn, H.: An algebraic condition for the approach to equilibrium of an open $N$-level system, Lett. Math. Phys. 2, 33 (1977). 